\title{
Transatlantica
}

Revue d'études américaines. American Studies Journal

\section{Jerome David Salinger (1919-2010) : l'ombre et la lumière}

\section{François Happe}

\section{(2) OpenEdition}

1 Journals

\section{Édition électronique}

URL : https://journals.openedition.org/transatlantica/4784

DOI : $10.4000 /$ transatlantica.4784

ISSN : 1765-2766

Éditeur

Association française d'Etudes Américaines (AFEA)

\section{Référence électronique}

François Happe, « Jerome David Salinger (1919-2010) : l'ombre et la lumière », Transatlantica [En ligne], 1 | 2010, mis en ligne le 29 septembre 2010, consulté le 10 février 2023. URL : http:// journals.openedition.org/transatlantica/4784; DOI : https://doi.org/10.4000/transatlantica.4784

Ce document a été généré automatiquement le 10 février 2023.

\section{(c) (i) (9)}

Creative Commons - Attribution - Pas d'Utilisation Commerciale - Pas de Modification 4.0 International - CC BY-NC-ND 4.0

https://creativecommons.org/licenses/by-nc-nd/4.0/ 


\title{
Jerome David Salinger (1919-2010) : l'ombre et la lumière
}

\author{
François Happe
}

1 Jerome David Salinger est mort le 27 janvier 2010, à l'âge de 91 ans - et à la grande surprise de ceux, nombreux, qui croyaient qu'il n'était déjà plus de ce monde.

2 Mais l'était-il encore vraiment ? Il faut dire qu'il avait cessé de publier après 1965 et n'écrivait plus, dit-on, que pour son propre plaisir (l'écriture, acte intransitif?). Sa première nouvelle, "The Young Folks » paraît en 1940 et la dernière, « Hapworth 16, 1924 », est livrée au public en 1965. Son mutisme dépasse ainsi de beaucoup sa carrière d'écrivain, si l'on s'en tient aux textes publiés à ce jour. Ironiquement, sa mort pourrait bien mettre un terme à ce long silence en forme de point d'interrogation. Cela fait en effet quarante-cinq ans que l'un des écrivains les plus adulés de l'histoire littéraire américaine a choisi de ne plus communiquer avec ses lecteurs - quarante-cinq ans que l'auteur de The Catcher in the Rye, le roman pour lequel l'expression « œuvre culte " aurait pu être inventée, s'acharne à se faire oublier.

3 En vain. La presse internationale a abondamment commenté l'événement. Je ne reproduirai pas ici le modèle de tous ces articles en deux parties, où un bref résumé de la vie de l'auteur disparu est suivi d'un rapide survol de l'œuvre (avec une mention spéciale pour le légendaire The Catcher in the Rye). Évitant le convenu des louanges consensuelles déversées en pareil cas, je souhaiterais, avant d'évoquer le silence de Salinger, revenir brièvement sur tous ces hommages parus après la disparition d'un écrivain qui, indiscutablement, occupe une place à part dans la littérature américaine. Et dont le talent avait effectivement de quoi rendre envieux plus d'un Bret Easton Ellis ${ }^{1}$.

4 À lire et à entendre ces nécrologies, ainsi que les réactions à ces dernières, il est frappant de constater que le lecteur, professionnel ou "amateur ", associe souvent son commentaire à la période de sa vie au cours de laquelle il a découvert les textes de Salinger - généralement The Catcher in the Rye. Le fait mérite d'être souligné dans la mesure où le jugement porté sur le livre se teinte fatalement d'une certaine nostalgie qui semble inhérente au rapport que l'on peut établir avec cette œuvre. Cette nostalgie, en d'autres termes, adhère au roman comme le parasite croît sur son hôte, au 
détriment de celui-ci. Il y a là, me semble-t-il, quelque chose d'injuste pour l'auteur qui se voit ainsi condamné, ou réduit, à être "l'écrivain de notre adolescence " comme on peut le lire fréquemment ${ }^{2}$. Il est permis de se demander, en effet, si l'enthousiasme et l'affection exprimés doivent être attribués à l'œuvre proprement dite ou à la tendresse que l'on garde pour tout ce qui reste attaché à notre jeunesse. Tendresse, ou myopie du souvenir, qui empêche parfois de voir des détails qui sont en fait un peu plus que cela. Il est vrai que les thèmes du roman prêtent à ce genre de confusion sentimentale - ce qui mène à une autre forme d'injustice dans sa réception : Holden est d'abord perçu comme l'adolescent rebelle-type (dans lequel on se plaît à se reconnaître) et on se sent poussé à dire sa révolte en termes sociologiques, voire idéologiques. The Catcher in the Rye est une œuvre littéraire, et ce serait rendre un bel hommage à son auteur que de débarrasser le roman des oripeaux qui en ont fait « un manuel pour comprendre les ados ».

Même lorsque l'approche se fait plus littéraire, on s'aperçoit que la tentation est grande de prendre Holden Caulfield pour l'héritier direct de Huckleberry Finn - en fait, je crois qu'on les confond même souvent - sans la moindre distance ironique. Le héros de Salinger n'est, assurément, pas avare de reproches à l'égard du monde adulte et de la société dans laquelle il vit, mais une attitude velléitaire n'est pas un engagement révolutionnaire, et les crimes les plus sérieux qu'il commet, consistent ni plus ni moins, à faire l'école buissonnière pendant trois jours et à tomber malade. (Et puis, il ne faut pas oublier que ce héros ne critique le monde que pour mieux le pardonner et proclamer, en fin de compte, son attachement à ce même monde). Chez Holden, ce n'est pas tant le personnage qui est intéressant, mais le narrateur. Ce qui invite l'analyse, ce n'est pas le jeune garçon que déprime le spectacle de son vieux professeur malade et fier d'exhiber sa couverture achetée à un Indien Navajo à Yellowstone, c'est l'instance qui reconstitue et raconte l'histoire. Pour reprendre la formule de Marc Chénetier, le véritable héros du livre, c'est le langage (Chénetier, 37). Si l'on admet le bien-fondé de cette observation, alors peut-être n'est-il plus utile de s'apitoyer avec Holden sur le sort des canards de Central Park quand le lac gèle en hiver - quoique je ne nie pas la portée métaphysique de l'intérêt du jeune homme pour les palmipèdes.

Il est un autre élément parasite dans la réception des textes de Salinger : c'est le choix qu'a fait l'auteur de vivre en reclus. Personne, semble-t-il, ne peut parler de l'écrivain sans insister sur le fait qu'il vivait à l'écart du monde - comme si cela conférait à son œuvre une valeur ajoutée. Bien sûr, comme le montre Don DeLillo, qui s'est en partie inspiré de J. D. Salinger pour composer le portrait de Bill Gray, l'écrivain reclus au nom couleur muraille de Mao II (1991), l'absence d'image, dans un univers de signes, est encore une image. L'invisibilité confère une aura qui fait de la personne célèbre une icône aussi sûrement qu'une surexposition médiatique:

When a writer doesn't show his face, he becomes a local symptom of God's famous reluctance to appear [...]. But we're all drawn to the idea of remoteness. A hard-to-reach place is necessarily beautiful. Beautiful and a little sacred maybe. And a person who becomes inaccessible has a grace and a wholeness the rest of us envy [...]. The writer who won't show his face is encroaching on holy turf. He's playing God's own trick (Delillo, 36-37).

7 Pour Salinger, comme pour le Bill Gray de DeLillo, l'isolement devait être synonyme d'intégrité et de liberté - cette liberté qui n'appartient qu'à ceux sur qui le monde n'a pas de prise. Mais c'est une liberté illusoire aussi, qui a privé les deux écrivains, le 
«vrai » comme le fictionnel, de toute prise sur le monde. À l'origine, Salinger s'était isolé, se retirant dans les bois de Cornish, dans le New Hampshire, pour écrire tranquillement ; or, malgré (ou serait-ce à cause de ?) cet isolement, il a fini par ne plus écrire du tout. Curieusement, c'est l'isolement que l'on retient d'abord et qui frappe l'imagination du public. Au lendemain de sa mort, la presse, dans son ensemble, saluait "Salinger le reclus » plutôt que « Salinger, l'écrivain qui s'était tu ». DeLillo le suggère, il y a un côté provocant dans le fait qu'une personne devienne inaccessible, et tout le monde se sent invité à avancer des raisons plausibles expliquant cet adieu au monde. Un écrivain se coupe de la société dans laquelle il vit? On s'empresse d'y voir une condamnation éclatante $d u$ système. Salinger contre l'argent de l'industrie du livre (comme le décrètent péremptoirement des analyses embryonnaires) : ne confondon pas l'auteur avec son personnage, Holden Caulfield ${ }^{3}$ ? Car s'il est un grand auteur, Salinger est aussi un phénomène de l'édition(The Catcher in the Rye se vend toujours à 250000 exemplaires par an), et c'est précisément cette industrie du livre qui lui a donné les moyens matériels de ne plus rien publier après 1965.

Si la retraite - au sens de l'éloignement - de Salinger suscite remarques et commentaires révérencieux (DeLillo a vu juste : « a grace and a wholeness the rest of us envy »), peu de voix s'élèvent pour tenter d'expliquer son silence, hormis, bien sûr, celles qui évoquent sa haine des grandes sociétés d'édition - oubliant que l'auteur avait pour habitude de publier ses nouvelles d'abord dans le New Yorker, avant de les confier à un éditeur. Il faut savoir que Salinger était chez lui au New Yorker. (Certains ont prétendu que c'est le New Yorker qui a « fait » Salinger). Franny and Zooey est dédié à William Shawn, que Salinger appelle "my closest friend", et qui dirigea le célèbre magazine jusqu'en 1987. Si c'était le monde de l'édition qu'il voulait fuir, on ne voit pas très bien pourquoi Salinger n'a pas continué à publier ses histoires dans le New Yorker, au moins jusqu'au départ de William Shawn. À mon sens, l'important n'est pas de savoir pourquoi l'auteur s'est coupé du monde. D'ailleurs, s'est-il vraiment coupé du monde? Après tout, Salinger n'est pas Pynchon; il a vécu à Cornish de 1953 à 2010, au vu et au su de tous, ou presque; son lieu de résidence n'a jamais été un mystère. L'important n'est même pas de savoir s'il a continué à écrire (des rumeurs parlent d'étagères couvertes de manuscrits dans son bureau, d'autres, plus modestes mais aussi peu vraisemblables, évoquent deux romans dans le coffre-fort d'un éditeur). La seule question qui vaille d'être posée, c'est pourquoi a-t-il cessé de publier après la parution de "Hapworth 16, 1924 ", le 19 juin 1965 ? Sans prétendre donner l'explication définitive, je crois qu'il est possible de trouver diverses raisons à ce silence, et c'est dans l'œuvre, dans la pratique d'écriture et dans le rapport particulier qui existe entre l'auteur et sa création qu'il convient de chercher.

Pour qui lit l'ensemble des textes publiés, le silence de Salinger peut apparaitre comme une conséquence prévisible de son évolution ${ }^{4}$. Tout d'abord, Salinger n'a jamais été un écrivain prolixe. Si sa production s'étale sur vingt-cinq ans, elle reste somme toute, plutôt modeste: un seul roman (The Catcher in the Rye, 1951), un recueil de neuf nouvelles (Nine Stories, 1953) dont sept avaient été d'abord publiées dans le New Yorker entre 1948 et 1953, deux volumes reprenant chacun deux longues nouvelles (Franny and Zooey, 1961, et Raise High the Roof Beam, Carpenters and Seymour an Introduction, 1963) toutes également publiées dans le New Yorker entre 1955 et 1959, et puis une dernière nouvelle, "Hapworth 16, 1924 ». Si on souhaite comprendre la genèse de l'œuvre, il convient d'ajouter la vingtaine de nouvelles publiées dans des magazines divers entre 
1940 et 1948, dont Salinger a interdit toute reproduction - mais dont il existe une édition pirate.

Relativement restreinte dans son volume, cette œuvre présente par ailleurs une texture événementielle ténue : il ne se passe pratiquement rien en termes d'intrigue. Seules les deux nouvelles qui bornent le recueil Nine Stories («A Perfect Day for Bananafish » et «Teddy ») se terminent par un événement tragique, la mort des deux protagonistes. Salinger n'est pas, au sens traditionnel de l'expression, un raconteur d'histoires, et luimême fait référence à plusieurs reprises au manque de fertilité de son imagination et répète qu'il ne se sent capable de produire que des textes courts. Cette affirmation deviendra d'ailleurs de moins en moins exacte au fil du temps, puisque toutes ses dernières nouvelles sur la famille Glass (de 1955 à 1965) sont particulièrement longues, la dernière, « Hapworth 16, 1924 » approchant les soixante-dix pages.

11 S'il a voulu plonger dans l'oubli les nouvelles de ses débuts, c'est évidemment parce qu'il les jugeait moins bonnes, mais c'est aussi parce que beaucoup d'entre elles peuvent être considérées comme des ébauches qu'il a réutilisées dans des textes plus aboutis. Son unique roman fait figure d'accident, ou, plus exactement, on peut dire que d'une certaine façon, son roman n'en est pas véritablement un : The Catcher in the Rye naît de la réunion de deux anciennes nouvelles, "I'm Crazy» (1945) et "Slight Rebellion off Madison» (1946), mettant en scène le protagoniste Holden Caulfield et fournissant ce qui constitue le cœur de l'histoire : le renvoi de Holden de son école et sa décision de passer trois jours à New York en attendant les vacances. Ce n'est pas l'intrigue, quasiment inexistante, qui tient le roman et en assure l'unité, mais la simple présence du protagoniste, et plus particulièrement, sa voix. Salinger ne construit pas ses histoires autour d'événements, mais autour de ses personnages et des relations qu'ils établissent entre eux - ou ne parviennent pas à établir, puisque la difficulté de communiquer est un thème majeur chez Salinger. Le moins que l'on puisse dire, c'est que le matériau diégétique mis en œuvre par l'auteur dans ses textes ne frappe pas par son foisonnement. L'écriture est pour lui un processus lent et douloureux. Sur la quatrième de couverture de Franny and Zooey, qui constitue, en dehors des textes euxmêmes, l'un des rares messages adressés par l'auteur à son lecteur, Salinger écrit, non sans ironie : «I work like greased lightning myself, but my alter-ego and collaborator, Buddy Glass, is insufferably slow ». Au milieu des années 1950, il travaillait quatorze ou seize heures par jour, souvent toute la nuit, même, détruisant plus qu'il ne conservait, exigeant des conditions de calme et de tranquillité extrêmes. À la naissance de sa fille, il installe son bureau dans une cabane à quelques centaines de mètres de la petite maison de Cornish, déjà très isolée dans la campagne du New Hampshire. Il lui arrive même de partir loin de chez lui, de se terrer dans un hôtel pour terminer une nouvelle. Comment s'étonner, dès lors, que tout échec - lorsqu'il estime que son travail n'est, finalement, pas publiable - soit suivi d'une période de doute, voire de dépression? L'angoisse de la création littéraire (et le jugement implacable qu'il porte sur sa production en fonction de ses propres critères) se double d'une quête mystique constante, la recherche d'une illumination qu'il conçoit comme consubstantielle à l'écriture. Bouddhisme Zen, Hindouisme, Vedanta, Christianisme, Christian Science, Scientologie... : les gourous, les guides, les systèmes de croyance se succèdent, aucun ne lui apportant la sérénité. Salinger n'en fait pas une question de religion au sens strict, associant philosophies ou pratiques orientales et christianisme. Pour autant, si la parabole de la Grosse Dame - symbole de l'humanité souffrante et répugnante et qui ne serait autre que Jésus Christ lui-même - est d'abord une invention de Zooey, je ne 
suis pas sûr de pouvoir déceler, entre l'auteur et le monde qu'il a créé, la moindre mise à distance.

12 À la difficulté d'écrire, qui ne fait que croître avec les années, il convient d'ajouter la sensibilité exacerbée de l'auteur à l'égard de ses textes, sensibilité qui semble indissociable des rapports extrêmement particuliers qu'il entretient avec son univers fictionnel. Après la publication de The Catcher in the Rye, il va se réfugier quelque temps en Angleterre. Pour lui, publier, c'est s'exposer, se mettre à nu. Ainsi que l'indique le début de sa dédicace de Raise High the Roof Beam, Carpenters and Seymour an Introduction ( If there is an amateur reader still left in the world-or anybody who just reads and runs... »), il veut bien confier ses textes aux lecteurs, mais il ne supporte pas l'idée que des critiques vont les analyser, les disséquer, émettre des jugements sur eux. D'aucuns affirment que c'est Mary McCarthy, avec son essai (très) critique sur Franny and Zooey, qui serait responsable de la décision d'un Salinger ulcéré de ne plus publier; mais il faudrait aussi évoquer les violentes attaques de Norman Mailer. Et de George Steiner. Et de Leslie Fiedler... Le manichéisme de Salinger et le nombrilisme de ses enfants prodiges en agaçaient plus d'un. Après avoir tant désiré la gloire, l'auteur de « For Esmé -with Love and Squalor » s'aperçoit (un peu tard) qu'il ne supporte pas d'être mis en cause publiquement. Plus que par les critiques adressées à l'écrivain, Salinger est profondément blessé par les attaques contre la famille Glass, qu'il aime d'amour. Que l'on ne s'y trompe pas, cela va bien au-delà de la tendresse qu'un auteur peut éprouver pour ses personnages. On a l'impression que pour Salinger, il existe une sorte de continuité entre la fiction et le monde réel, ses protagonistes ne sont pas que des créatures linguistiques, ils «vivent» vraiment. Cela explique, par exemple, qu'il ne peut se résoudre à ne leur accorder qu'une existence éphémère, l'espace d'une nouvelle, pour les remplacer par d'autres dans l'histoire suivante. C'est ainsi que les mêmes personnages réapparaissent d'une nouvelle à l'autre, des familles entières se constituent - la fiction de Salinger, c'est d'abord une histoire de famille(s): les Gladwaller et les Caulfield, préfigurant la fameuse tribu des Glass. Le sergent Babe Gladwaller (" Last Day of the Last Furlough », « A Boy in France ", " The Stranger »), qui doit lui-même beaucoup au sergent Salinger, réapparait sous les traits du sergent $X$ dans "For Esmé-with Love and Squalor", et on peut fort bien imaginer que cet anonymat ne masque qu'imparfaitement Seymour Glass lui-même. La petite sœur de Babe Gladwaller, Mattie, également présente dans les trois nouvelles citées, deviendra la Phoebe des nouvelles où apparaît Holden, et donc celle de The Catcher in the Rye, mais elle est aussi Sybil dans " A Perfect Day for Bananafish ». Le Grand Frère Mort, la Petite Sœur au Cœur Pur, l'Adolescent Rebelle - pour ne citer que les plus importants constituent ainsi une sorte de galerie de personnages récurrents, une constellation dans l'univers de Salinger. Ils n'habitent pas seulement sa fiction, ils vivent avec lui. Ou lui avec eux. Lorsque Salinger refuse de vendre les droits de son roman à Hollywood, il ajoute, en guise d'explication: «Holden wouldn't like it». Et ce n'est pas une simple boutade. À une étudiante l'interrogeant sur sa vie (à une époque où il communiquait encore avec le monde extérieur), il répond que tout est dans ses textes. Lorsque Teddy, dans la nouvelle éponyme, déclare "I met a lady, and I sort of stopped meditating " (Salinger, Nine Stories, 188), Salinger fait référence à sa rencontre avec Claire Douglas, qu'il épousera en 1955 : lorsqu'il la voit pour la première fois, en 1950, elle a seize ans, il en a trente-et-un. Elle fréquente Shipley School, comme Jane Gallagher dans The Catcher. Il tombe sous le charme de la jeune fille, mais à l'époque il songe sérieusement à devenir moine bouddhiste. Le mariage et la sexualité sont pour lui des souillures 
difficilement compatibles avec la méditation. Le corps et ses fonctions biologiques seront un problème pour Salinger tout au long de sa vie. Comme le Sergent X de «For Esmé » et comme Seymour Glass, Salinger souffre de dépression à la fin de la guerre et passera quelque temps dans un hôpital militaire. Et toute la fiction de Salinger fourmille de ces données empruntées à sa propre expérience. Bien évidemment, je ne suggère pas ici qu'il convient d'analyser l'œuvre à la lumière d'éléments biographiques (qui d'ailleurs n'expliquent rien), je conçois plutôt cette relation osmotique entre la vie et l'œuvre comme un symptôme. D'ailleurs, contrairement à ce qui se passe dans une démarche autobiographique, ce n'est pas la vie qui envahit la fiction, ce serait plutôt l'inverse. Avec l'arrivée des Glass, Salinger s'acharne à faire tomber les barrières ontologiques qui le séparent de «sa » famille : « I've been waiting for them most of my life ", ajoute-t-il sur la quatrième de couverture de Franny and Zooey, comme pour parler de parents enfin (re)trouvés. Seymour, c'était Salinger avec son expérience traumatisante de la guerre ; Salinger devient de plus en plus Seymour. Seulement voilà, Seymour, le poète, le saint, le prodige, se suicide lors de sa première apparition, alors Salinger lui invente une vie à rebours et, pour les besoins de ce projet hagiographique, crée son alter ego, Buddy Glass, le frère et le double de Seymour : «Is it so bad that we sometimes sound like each other? The membrane is so thin between us " (Salinger, Raise High the Roof Beam, Carpenters andSeymour an Introduction, 159), lui écrit le grand frère absent dans une vieille lettre. Car à mesure que Salinger se fond dans les Glass, son écriture se transforme. L'oralité du roman, les dialogues de Nine Stories, disparaissent au profit de longs monologues, d'extraits de journaux, de lettres, seuls moyens de faire "revivre " le disparu, jusqu'à cette très improbable et interminable lettre que le jeune Seymour, alors âgé de sept ans, envoie à sa famille depuis son camp de vacances, « Hapworth 16, 1924 », dernière missive de J. D. Salinger à son lecteur.

13 La prose se fait dense, la parole proliférante et le récit, dédale de digressions. Buddy nous avait prévenus: "[Seymour's] character lends itself to no legitimate sort of narrative compactness that I know of [...]. I want to distribute mementos, amulets, I want to break out my wallet and pass around snapshots " (Salinger, Raise High the Roof Beam, Carpenters andSeymour an Introduction, 106-107). La dissolution des formes mériterait une étude spécifique, mais on voit bien où Salinger nous conduit: il veut nous faire partager ces photos de famille, nous faire entrer dans l'intimité des Glass ( Zooey» se passe en grande partie dans la salle de bains du personnage central, le lecteur est invité à suivre Franny dans les toilettes d'un restaurant). Bien sûr, la dissolution des formes n'est autre que l'invention d'une autre forme; bien sûr, les digressions de Buddy, les photos de famille qu'il fait circuler, les textes qui tournent autour d'un centre vide et s'acharnent à faire surgir l'absent constituent aussi, et peutêtre surtout, la mise en scène d'une profonde et très émouvante réflexion sur l'écriture. Il n'empêche que le lecteur " amateur » est en droit de se dire que ses rapports avec les Glass relèvent autant de l'acte de foi que de l'acte de lecture. Bien sûr, l'intention de Salinger est toute autre, il n'empêche, ce même lecteur " amateur " peut s'imaginer que les nouvelles sur les Glass invitent plus à la constitution d'un fan-club que d'un lectorat. Évoquant sa famille de fiction (toujours sur cette fameuse quatrième de couverture de Franny and Zooey), Salinger déclare, peut-être avec une lucidité qui s'avérera prophétique : «It is a long-term project, patently an ambitious one, and there is a realenough danger, I suppose, that sooner or later I'll bog down, perhaps disappear entirely, in my own methods, locutions, and mannerisms ». Il se lance à la recherche de cette famille un peu comme Proust se lance à la recherche du temps perdu. Toutes 
proportions gardées, il y a en effet quelque chose de proustien dans cette exploration de la mémoire du narrateur, Buddy Glass, qui n'est que le prétexte à une réflexion sur l'écriture. Et le rapprochement ne doit rien au hasard. Dans "Hapworth 16, 1924 ", Seymour nous livre peut-être l'une des clés du mystère que constitue le silence de Salinger. Dans cette lettre, qui prend parfois des allures de catalogue de la section "Littérature" d'une grande bibliothèque, le jeune garçon cite, en français, naturellement, une phrase de Proust: «On ne trouve jamais aussi hauts qu'on avait espérés, une cathédrale, une vague dans la tempête, le bond d'un danseur " (Salinger, «Hapworth 16, $1924 »$, 96). Cette remarquable expression de l'attente déçue, empruntée au deuxième tome de la Recherche, À l'ombre des jeunes filles en fleurs, où le narrateur explique que le cérémonial précédant l'arrivée de Mme Swann suscitait tant de promesses dans son imagination que l'apparition finale n'était jamais à la hauteur de son attente, résonne bien étrangement, placée ainsi, à quelques pages de la fin de la dernière nouvelle. (Lorsque paraît ce long texte dans le New Yorker en 1965, cela fait déjà six ans que Salinger n'a rien publié).

L'arrivée, décevante, de Mme Swann serait-elle la métaphore du projet ambitieux dont parlait Salinger, le reflet de cette famille Glass qu'il a attendue, nous dit-il, la plus grande partie de sa vie?

Seymour, qui est aussi la conscience de Buddy, lui avait écrit, dans une lettre :

[D]o you know what you will be asked when you die? But let me tell you first what you won't be asked. You won't be asked if you were working on a wonderful, moving piece of writing when you died [...]. I'm so sure you will be asked only two questions. Were most of your stars out? Were you busy writing your heart out? (Salinger, Raise High the Roof Beam, Carpenters and Seymour an Introduction, 160).

Quelle que soit la distance qui sépare Buddy, l'écrivain fictionnel, de Salinger, l'écrivain "réel », il ne fait guère de doute que ce dernier a bien essayé d'éteindre toutes ses étoiles. Il est aussi possible qu'à force d'écrire ce que lui dictait son cœur, il se soit embourbé dans ses propres excès - comme il l'avait envisagé -, mais le risque de l'excès n'est-il pas inhérent à toute entreprise artistique, ainsi que l'écrivait John Updike en 1961, dans son commentaire sur Franny and Zooey: «[T]he refusal to rest content, the willingness to risk excess on behalf of one's obsessions, is what distinguishes artists from entertainers, and what makes some artists adventurers on behalf of us all » (Grunwald, Salinger, 56) ?

Lorsqu'il parle du bonheur de ne pas publier, l'auteur de « Hapworth » fait référence au soulagement de ne plus subir cette pression qu'il s'est toujours imposé à lui-même. J. D. Salinger s'est peut-être tu tout simplement parce qu'il craignait de ne plus pouvoir écrire une œuvre à la mesure de son ambition. 


\section{BIBLIOGRAPHIE}

CHÉNETIER, Marc, Au-delà du soupçon : La nouvelle fiction américaine de 1960 à nos jours, Paris, Seuil, 1989.

DELILLO, Don, Mao II, London, Vintage, 1992.

GRUNWALD, Henry Anatole, ed. Salinger, A Critical and Personal Portrait, New York, Harper Colophon Books, 1963.

MATHÉ, Sylvie, dir. Profils Américains :J.D. Salinger, Montpellier, Publications Montpellier 3, 2001.

SALINGER, Jerome David, The Catcher in the Rye, Harmondsworth, Penguin, 1978.

SALINGER, Jerome David, Nine Stories, New York, Bantam, 1964.

SALINGER, Jerome David, Raise High the Roof Beam, Carpenters and Seymour, an Introduction, New York, Bantam, 1965.

SALINGER, Jerome David, Franny and Zooey, New York, Bantam, 1966.

SALINGER, Jerome David, « Hapworth 16, 1924 », in The New Yorker, XXXXI, June 19, 1965, 32-113.

SALINGER Margaret A, Dream Catcher, New York, Washington Square Press, 2000.

\section{NOTES}

1. Peu après l'annonce de la mort de Salinger, l'auteur de American Psycho a fait paraître sur son compte Twitter le commentaire suivant : «Yeah!! Thank God he's finally dead. I've been waiting for this day for-fucking-ever. Party tonight!!! ». J'imagine qu'il en va de cette déclaration comme de tout ce qu'écrit Ellis : il ne faut pas vraiment la prendre au sérieux.

2. J'ai bien conscience qu'il existe des raisons objectives au fait que la découverte de certains textes corresponde à une certaine époque de la vie, mais combien d'auteurs restent, comme Salinger, étroitement associés à cette période bien précise ?

3. À New York, il n'y a que l'argent qui parle, nous affirme effectivement Holden, à l'aise dans son manteau douillet, et confortablement calé dans sa petite vie bourgeoise, sans se demander si les pauvres auraient les mêmes pudeurs. Mais l'univers de Salinger est un monde où les fins de mois n'existent pas. Bien entendu, il ne s'agit en aucune manière de reprocher à l'écrivain d'avoir situé ses enjeux ailleurs que sur le terrain idéologique, je souhaite simplement souligner que l'on ne saurait faire de Salinger un chevalier blanc pourfendant le système capitaliste tout entier.

4. Bien sûr, la prévision est toujours plus facile a posteriori, lorsque les faits sont déjà venus confirmer ce que l'on se propose de "prévoir", mais lorsque j'évoque une "dérive vers le silence » en 2001 (Mathé, 19), je reprends une idée que j'avais déjà avancée dans des travaux consacrés à Salinger en 1978. Depuis cette époque, il m'a toujours paru peu probable que Salinger puisse publier de nouveaux textes, et aujourd'hui encore, je reste sceptique quant à l'existence de tous ces prétendus manuscrits - même si on ne peut pas exclure une opération semblable à celle du manuscrit inachevé de Nabokov. 
INDEX

Thèmes : Reconnaissances

\section{AUTEUR}

FRANÇOIS HAPPE

Université d'Orléans 